\title{
Fatigue Analysis of Notched Laminates: A Time-Efficient Macro-Mechanical Approach
}

\author{
P. Naghipour ${ }^{1}$, E. J. Pineda ${ }^{2}$, B.A. Bednarcyk ${ }^{2}$, S. M. Arnold ${ }^{2}$, A. M. Waas ${ }^{3}$ \\ 1) Ohio Aerospace Institute, Cleveland, $\mathrm{OH}, 44142$ \\ 2) NASA Glenn Research Center, Cleveland, $\mathrm{OH}, 44135$ \\ 3) University of Washington, Seattle, WA 98195
}

\begin{abstract}
A coupled transversely isotropic deformation and damage fatigue model is implemented within the finite element method and was utilized along with a static progressive damage model to predict the fatigue life, stiffness degradation as a function of number of cycles, and postfatigue tension and compression response of notched, multidirectional laminates. Initially, the material parameters for the fatigue model were obtained utilizing micromechanics simulations and the provided [0], [90] and $[ \pm 45]$ experimental composite laminate $S-N$ data. Within the fatigue damage model, the transverse and shear properties of the plies were degraded with an isotropic scalar damage variable. The damage in the longitudinal (fiber) ply direction was suppressed, and only the strength of the fiber was degraded as a function of fatigue cycles. A maximum strain criterion was used to capture the failure in each element, and once this criterion was satisfied, the longitudinal stiffness of the element was decreased by a factor of $10^{4}$. The resulting, degraded properties were then used to calculate the new stress state. This procedure was repeated until final failure of the composite laminate was achieved or a specified number of cycles reached. For post-fatigue tension and compression behavior, four internal state variables were used to control the damage and failure. The predictive capability of the abovementioned approach was assessed by performing blind predictions of the notched multidirectional IM7/977-3 composite laminates response under fatigue and post-fatigue tensile and compressive loading, followed by a recalibration phase. Although three different multidirectional laminates were analyzed in the course of this study, only detailed results (i.e., stiffness degradation and post-fatigue stress-strain curves as well as damage evolution states for a single laminate ([30/60/90/-30/-60 $\left.]_{2 s}\right)$ are discussed in detail here.
\end{abstract}

\section{Introduction}

The coupled transversely isotropic deformation and damage fatigue model [1-5] known as ADEAL (Anisotropic Damage Evolution and Life) employs a scalar damage variable, which evolves with the number of cycles (See Figure 1). One advantage of this fatigue model is its ability to be integrate with a cyclic jumping scheme. For the present work, a single load cycle is applied and the number of additional cycles required to damage the material by an additional amount equal to the specified damage increment is calculated per element. The controlling element (minimum number of cycles) number of cycles is then used for all elements to determine the amount of damage in every element for $\mathrm{N}$ cycles. Within the ADEAL model, an isotropic scalar damage variable is used to degrade the transverse and shear properties of all the plies. The longitudinal stiffness degradation is assumed to be minimal and only the strength of the fiber is degraded due to interfacial wear as a function of fatigue cycles. A logarithmic degradation function is used for the critical stress/strain of the fiber in the longitudinal direction. Once this critical stress/strain was reached, the longitudinal (E11) stiffness of the element was decreased by a factor of $10^{4}$. The stress-strain histories are updated with the new damage level and the process is repeated until complete failure or a certain number of cycles is reached. 
The Enhanced Schapery Theory (EST) progressive damage model (used for post-fatigue tension and compression behavior) utilizes four internal state variables (ISVs) to control the damage and failure degradation. All damage is said to result from matrix microdamage, controlled by a single ISV, which degrades the transverse and shear moduli of the lamina. Three separate failure ISVs are used to incorporate failure due to fiber breakage, mode I matrix cracking, and mode II matrix cracking [6].

Three different notched quasi-isotropic IM7/977-3 multidirectional laminates; Layup 1: $[0,45,90,-45]_{2 S}$, Layup $2[+60,0,-60]_{3 s}$ and Layup 3: $[+30,+60,90,-60,-30]_{2 s}$ were analyzed as part of the Tech-Scout 1 project. Fatigue response of these composite laminates along with post-fatigue tensile and compressive behaviour was first predicted and then recalibrated using the above-mentioned ADEAL and EST models. However, only the results for Layup $3\left([30 / 60 / 90 /-30 /-60]_{2 s}\right)$ will be documented here in detail.

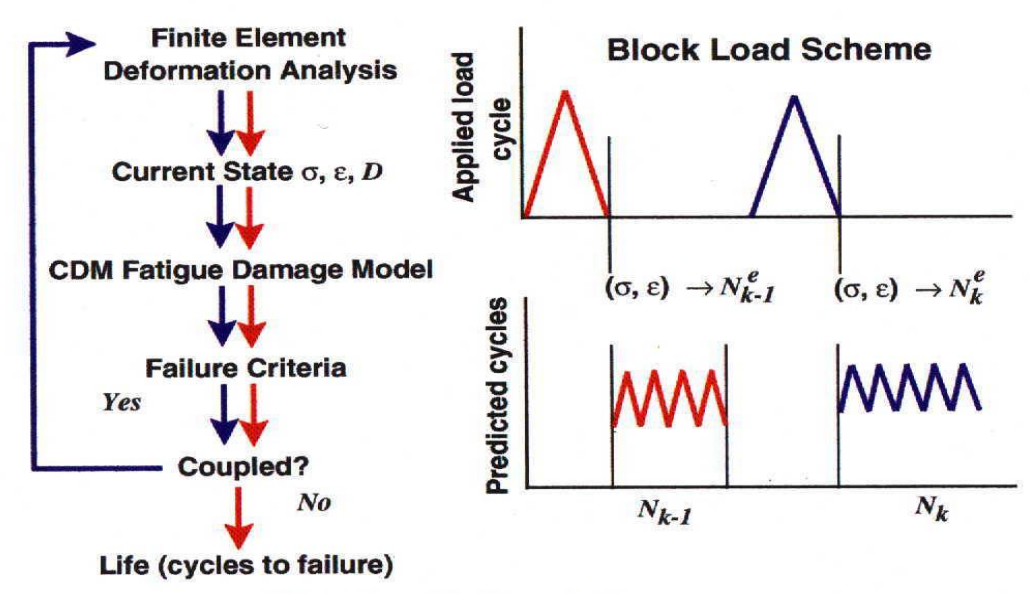

Life Prediction Scheme

Figure 1: Coupled deformation and damage methodology

\section{Characterization and Calibration}

The implementation of the ADEAL model within NASA Glenn Research Center's Micromechanics Analysis Code with Generalized Method of Cells (MAC/GMC) [7] was used, along with the code's classical lamination theory. Unnotched [0], [90], and $[ \pm 45]_{4 s}$ coupon experimental S-N data were provided by the AFRL Tech Scout Project [8] and were used to calibrate the material parameters for the ADEAL model. The micromechanics capabilities of MAC/GMC were not used herein because (i) the input coupon level data lacked the fidelity required to implement a theory at the fiber/matrix scale, and (ii) efficiency was critical as the analyses were on a tight turn-around schedule as part of the AFRL Tech Scout Project. The effective transversely isotropic, linear elastic, deformation properties and the EST macroscopic progressive damage model parameters utilized are those obtained during the prior static prediction phase of the study [8] (Table insert in Figure 2). The resulting ADEAL material parameters obtained are given in the table insert in Figure 2, along with the corresponding characterization results (solid lines) and experimental data (symbols). 


\begin{tabular}{|c|c|c|c|}
\hline $\mathrm{E}_{11}=164.3 \mathrm{GPa}$ (Tension/Compression) & $X_{11}^{\top}=0.0161$ & $e s 0=1.0\left(P^{-1 / 3}\right)$ & $g s 0=1.0\left(P^{-1 / 3}\right)$ \\
\hline$E_{22}=8.97 \mathrm{GPa}$ & $X_{11}{ }^{c}=0.0098$ & es1 $=-7.56 e-3$ & $g s 1=-5.58 e-3$ \\
\hline$v_{12}=0.3197$ & $Y_{22}{ }^{\top}=0.005$ & es $2=2.13 e-4$ & $g s 2=-5.84 e-5$ \\
\hline$G_{12}=4.88 \mathrm{GPa}$ & $X_{22}{ }^{C}=0.0311$ & $e s 3=-3.62 e-6$ & $g s 3=7.07 e-7$ \\
\hline$G_{1}{ }^{T / \text { Fiber }}=40 / G_{1}{ }^{C / \text { Fiber }}=10(\mathrm{~N} / \mathrm{mm})$ & $S_{12}=0.036$ & es $4=2.41 e-8$ & $g s 4=-2.49 e-9$ \\
\hline$G_{1}^{T / M a t r i x} / G_{1}{ }^{C / M a t r i x}=0.256(\mathrm{~N} / \mathrm{mm})--D C B$ & & $e s 5=5.64 e-11$ & $g s 5=2.70 e-12$ \\
\hline$G_{2}^{\text {Matrix }}=1.156(\mathrm{~N} / \mathrm{mm})-\mathrm{ENF}$ & & & \\
\hline
\end{tabular}

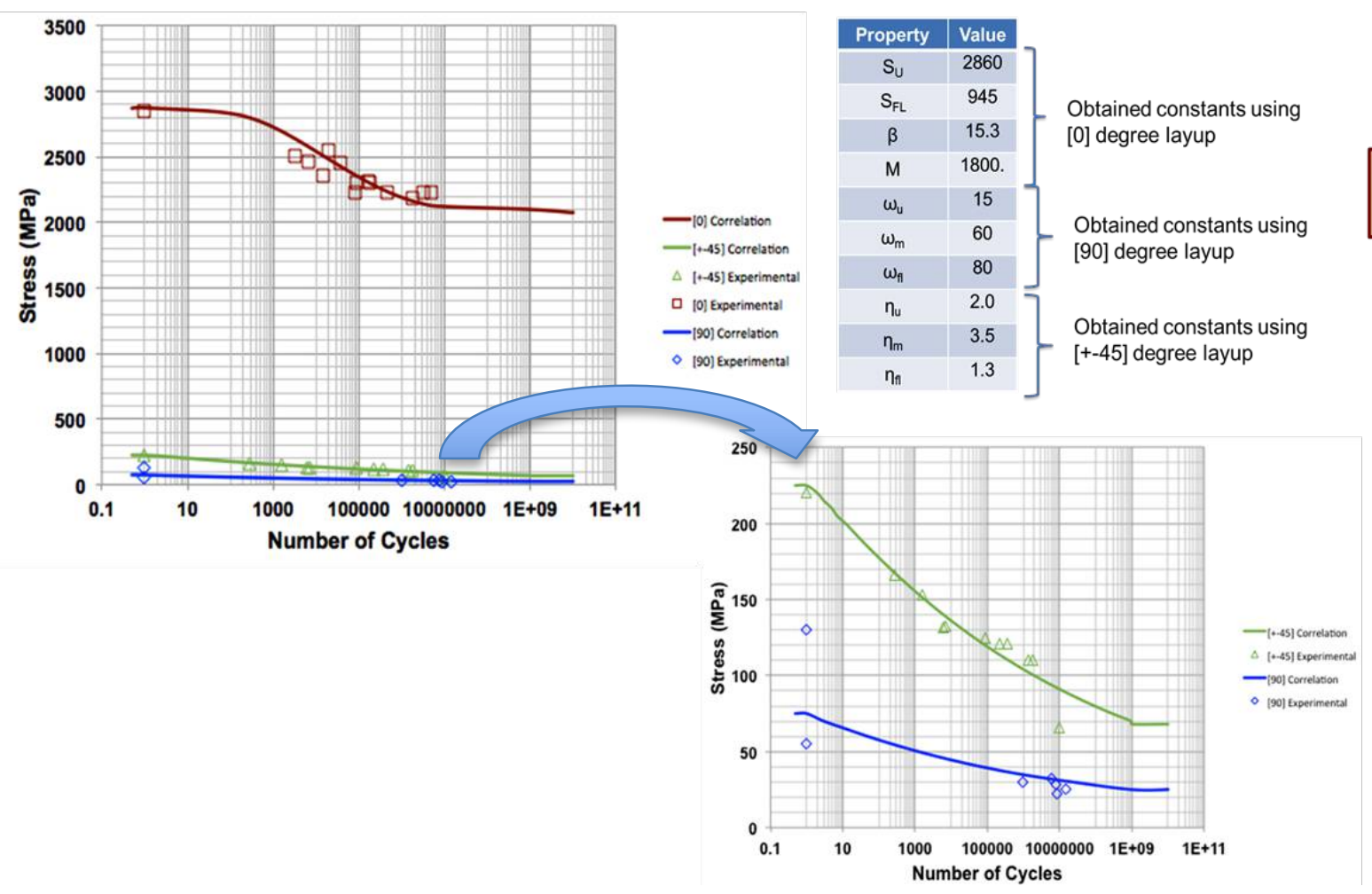

Figure. 2: Characterization of [0], [90], and [ \pm 45$]$ S-N curves: red [0] ply, blue is [90] and green is [ \pm 45$]$ laminates.

\section{Results and Discussion}

The simulation strategy for the notched specimens entailed modelling the multidirectional layups with $5,137,1 \mathrm{~mm}^{2}, 2 \mathrm{D}$ shell elements (S4R). Figure 3 shows the FEA mesh utilized. Traction boundary conditions were applied at one end of the specimen, whereas the other end was fixed in the loading direction. The transverse $\left(E_{22}\right)$ and shear $\left(G_{12}\right)$ properties of the plies were degraded, according to the ADEAL fatigue model, at the end of each load block. Furthermore, it was assumed that stiffness degradation in the fiber direction was minimal, thus damage in the longitudinal ply direction was supressed (i.e., $E_{11}$ not degraded with cycles) and it was assume that only the strength of the fiber was degraded because of wear as a function of fatigue cycles. An assumed degradation function was used for the critical strain of the fiber $\left(\varepsilon_{11}{ }^{c}\right)$

$$
\varepsilon_{11}^{C}=a \log _{10}(N)+b
$$


where $a=-0.0092$ and $b=0.063$ (for the blind predictions). The longitudinal strain in each element was also compared to the current longitudinal failure strain, if the criteria was met or exceeded the $E_{11}$ stiffness of the element was eliminated. The resulting, degraded properties were then used to calculate the new stress state during the next increment in cycles, applied within a single load block. This procedure was repeated until final failure of the composite laminate was achieved or a specified number of cycles reached.

Subsequent to the fatigue analysis, the final damaged state at a prescribed number of cycles is used as an initial state for residual tension and compression after fatigue simulations. EST was used to predict the post-fatigue strengths after $200 \mathrm{k}$ cycles as applied in the fatigue analysis.

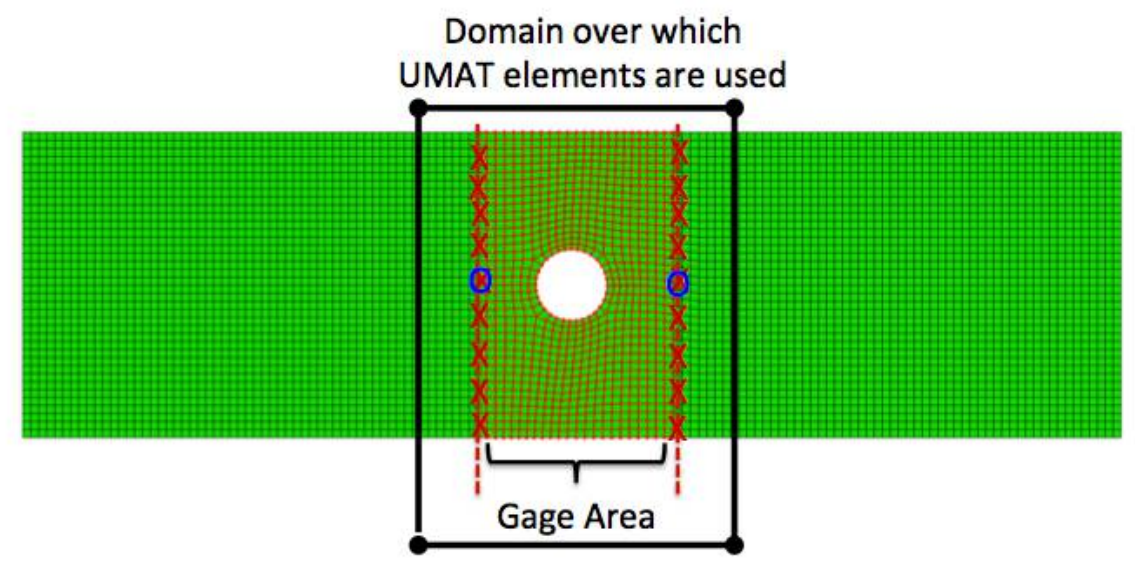

Figure 3: FEA mesh used for all layups and description of virtual extensometer

An error in processing simulated extensometer strain results (see Figure 3 ) was found subsequent to submittal of our blind predictions that resulted in incorrect global strain calculations, which in turn affected the initial (zero ${ }^{\text {th }}$ cycle) and subsequent reported cycle stiffnesses. This data processing error, is illustrated in Figure 3. Only a single node (above and below the hole - indicated by blue circles) was used to calculate strains in the originally reported results. However, a more realistic method to mimic the extensometer strain (as done in previous static deformation results [8]) is to use the average of the nodal displacements along the extensometer plane (indicated by the red $X^{\prime} s$ ) in the global strain calculation. This averaging process is necessary for consistency with previously reported deformation results and enables the corrected (measured) initial stiffness to be recovered. Utilizing only a single point in the presence of high deformation gradients is problematic and produces incorrect initial stiffness results. Original and reprocessed stiffness degradation with subsequent cycles and post-fatigue stress-strain behaviour for Layup 3 are demonstrated in Figure 4. Meanwhile, the predicted error in the residual stiffness, strength, and strain after $200 \mathrm{~K}$ cycles for Layup $3\left([+30,+60,90,-60,-30]_{2 s}\right.$ are given in Table 1.

\begin{tabular}{|l|c|c|c|c|c|c|c|c|c|}
\hline Layup 3 & $\begin{array}{c}\text { Stiffness } \\
(\mathrm{GPa}) \\
\text { Exp* }\end{array}$ & $\begin{array}{c}\text { Stiffness (GPa) } \\
\text { (Prediction) }\end{array}$ & $\begin{array}{c}\% \\
\text { Error }\end{array}$ & $\begin{array}{c}\text { Max stress } \\
(\mathrm{MPa}) \\
\text { Exp* }\end{array}$ & $\begin{array}{c}\text { Max stress } \\
\text { (MPa) } \\
\text { (Prediction) }\end{array}$ & $\begin{array}{c}\% \\
\text { Error }\end{array}$ & $\begin{array}{c}\text { Max } \\
\text { strain } \\
\text { Exp* }\end{array}$ & $\begin{array}{c}\text { Max strain } \\
\text { (Prediction) }\end{array}$ & $\begin{array}{c}\% \\
\text { Error }\end{array}$ \\
\hline OHT & 32.2 & 29.9 & 7.1 & 424 & 303 & 28.5 & 0.0147 & 0.0105 & 28.6 \\
\hline OHC & 32.9 & 29.9 & 9.1 & -274 & 165 & 39.8 & -0.011 & -0.006 & 45.4 \\
\hline
\end{tabular}


Table 1 Predicted stiffness, strength, and strain after a prescribed number of fatigue cycles at a given uniaxial load for Layup $3\left([+30,+60,90,-60,-30]_{2 s}\right.$ after 200,000 Cycles. (Exp*: Experiment)
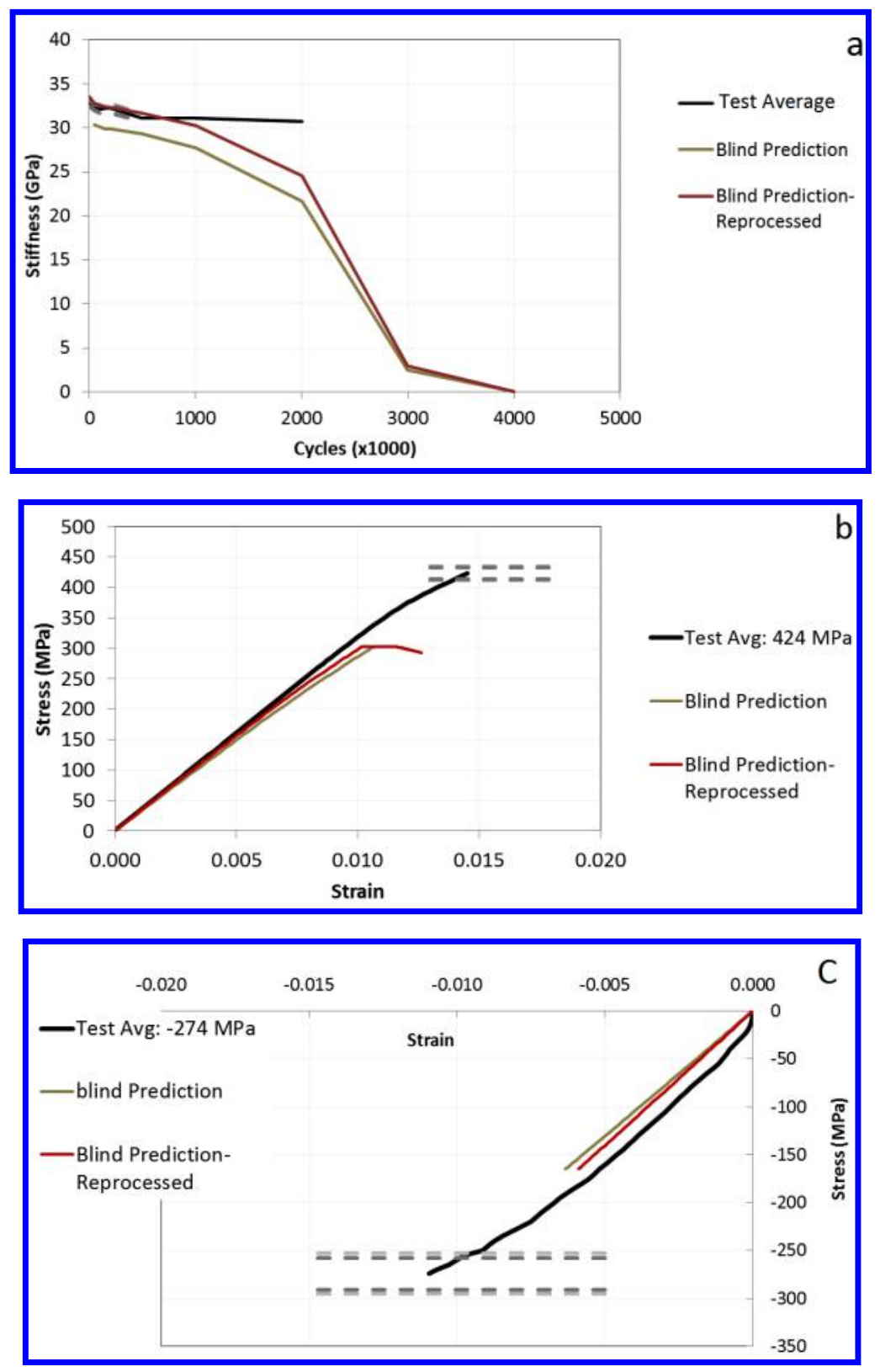

Figure 4: Layup $3[+30,+60,90,-60,-30]_{2 s}$ original and reprocessed blind predictions. a) Stiffness degradation as function of cycles, b) OHT residual strength after 200, 000 cycles, c) OHC residual strength after 200,000 cycles

In the recalibration phase of this study, no changes were made to any deformation or fatigue parameters, except for the assumed evolution of longitudinal strain (i.e. the fiber strain) to failure as a function of cycles (see Equation. 1). It was surmised that since i) Graphite fiber strength and ii) the cyclic longitudinal interfacial wear which gives rise to further strength reduction of the fiber, is highly volume sensitive; the effective longitudinal failure stress associated with a [0] laminate as a function of cycles should be significantly influenced by the volume of material within a given finite element. The same 
assumption can be made for strain since the deformation behaviour is assumed linear elastic. That is the relative damage length scale associated with a given analysis as compared with that length scale used for characterization must be accounted for. Consequently, we decided to scale the longitudinal UTS associated with the [0] unidirectional laminate fatigue response by the well-known Weibull volume fraction equation [9]:

$$
\sigma_{2} / \sigma_{1}=\left(A_{1} / A_{2}\right)^{(1 / m)}
$$

where $A_{1}$ is the original area assumed for characterization (i.e., the entire gage area of the specimen, which is equal to $25 \mathrm{~mm}$ by $12.5 \mathrm{~mm}$ ) and $A_{2}$ is the area associated with the size of the finite element used in the notched laminate analysis (i.e., $1 \mathrm{~mm}^{2}$ ). The Weibull parameter for the fiber strength degradation as a function of cycles, $\mathrm{m}$, was taken to be 10 based on previous COPV experience [10]. Figure 5 illustrates both the original unnotched [0] laminate $\mathrm{S}-\mathrm{N}$ curve given (and used) for characterization (see purple curve) and the scaled (black) [0] laminate S-N curve using Equation. (2). Note the fatigue limit of the [0] laminate remained unaltered since it was assumed that this lower limit (associated primarily with matrix damage of the polymer) would not be influenced greatly by the volume of material being analysed. Given this scaled S-N curve, Equation (1) was then adjusted, such that $a=-0.0025$ and $b=0.034$. Note that the effective [0] modulus, $E_{11}$, and longitudinal strain, $\epsilon_{11}^{c}$, was used to obtain the corresponding S-N curve from Equation. (1).

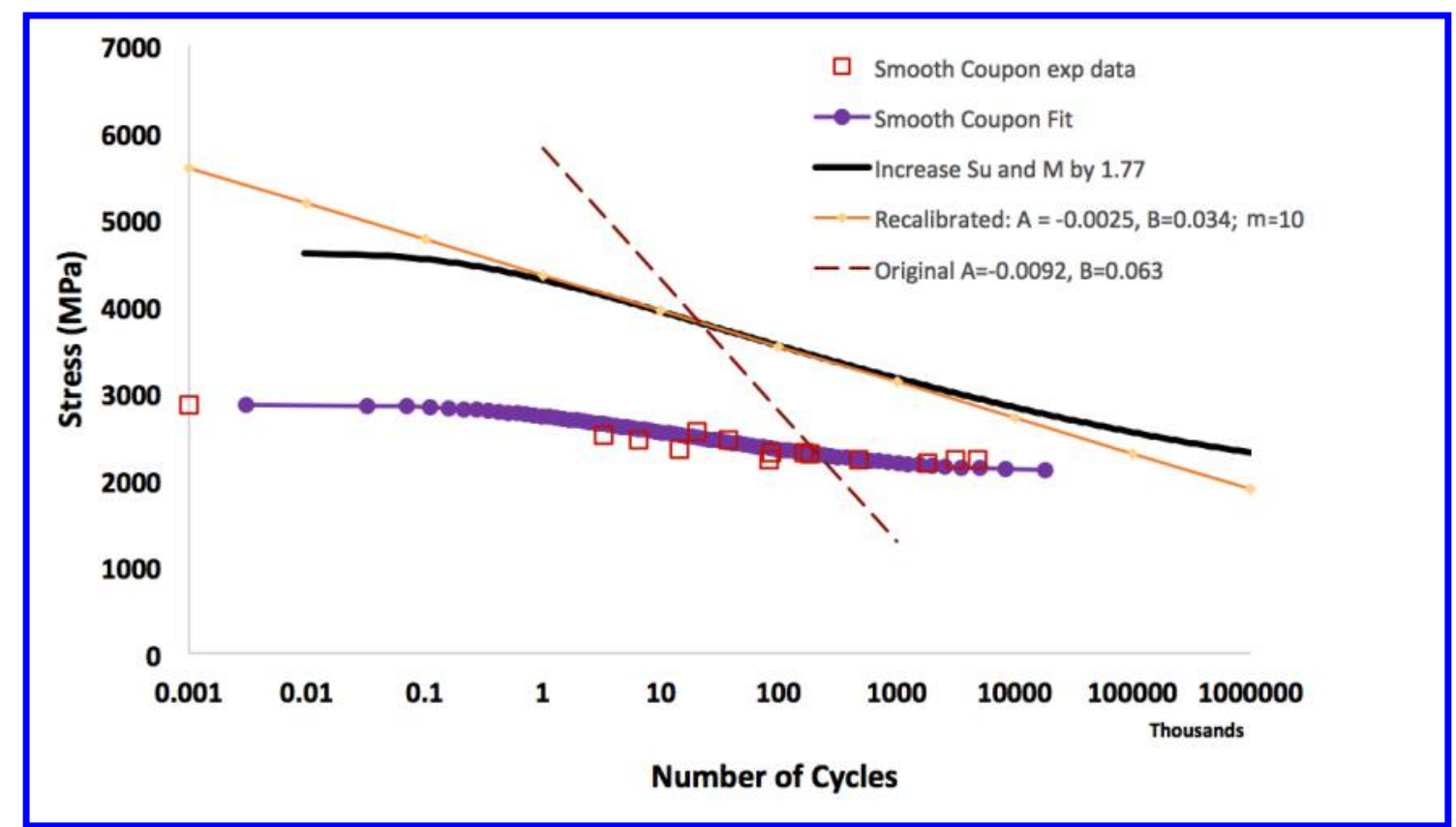

Figure 5: Modified Cyclic Dependence of effective longitudinal stress (i.e., fiber failure strain = $\mathrm{S} / \mathrm{E}_{11}$ ) based on the volume of the finite element.

Now given this new evolution of the critical strain to failure as a function of cycles in the longitudinal direction, all the fatigue analyses were performed again. The recalibration error in the residual stiffness, strength and strain after 200K cycles for Layup 3 are summarized in Table 2. Detailed results (effective laminate stiffness as a function of cycles in the loading direction and residual stress-strain response (i.e., strength) after a given number of cycles) for Layup 3 are also shown in Figure 6. 


\begin{tabular}{|l|c|c|c|c|c|c|c|c|c|}
\hline Layup 3 & $\begin{array}{c}\text { Stiffness } \\
(\mathrm{GPa}) \\
\text { Exp* }\end{array}$ & $\begin{array}{c}\text { Stiffness } \\
(\mathrm{GPa}) \\
\text { (Prediction) }\end{array}$ & $\begin{array}{c}\% \\
\text { Error }\end{array}$ & $\begin{array}{c}\text { Max stress } \\
(\mathrm{MPa}) \\
\text { Exp* }^{*}\end{array}$ & $\begin{array}{c}\text { Max stress } \\
(\mathrm{MPa}) \\
\text { (Prediction) }\end{array}$ & $\begin{array}{c}\% \\
\text { Error }\end{array}$ & $\begin{array}{c}\text { Max } \\
\text { strain } \\
\text { Exp* }\end{array}$ & $\begin{array}{c}\text { Max strain } \\
\text { (Prediction) }\end{array}$ & $\begin{array}{c}\% \\
\text { Error }\end{array}$ \\
\hline OHT & 32.2 & 32.5 & -0.93 & 424 & 392 & 7.6 & 0.0147 & 0.0141 & 4.1 \\
\hline OHC & 32.9 & 32.5 & 1.22 & -274 & -214 & 21.9 & -0.011 & -0.008 & 27.2 \\
\hline
\end{tabular}

Table 2 Recalibrated stiffness, strength, and strain after a prescribed number of fatigue cycles at a given uniaxial load for Layup $3\left([+30,+60,90,-60,-30]_{2 s}\right.$ after 200,000 Cycles. (Exp*:Experiment)
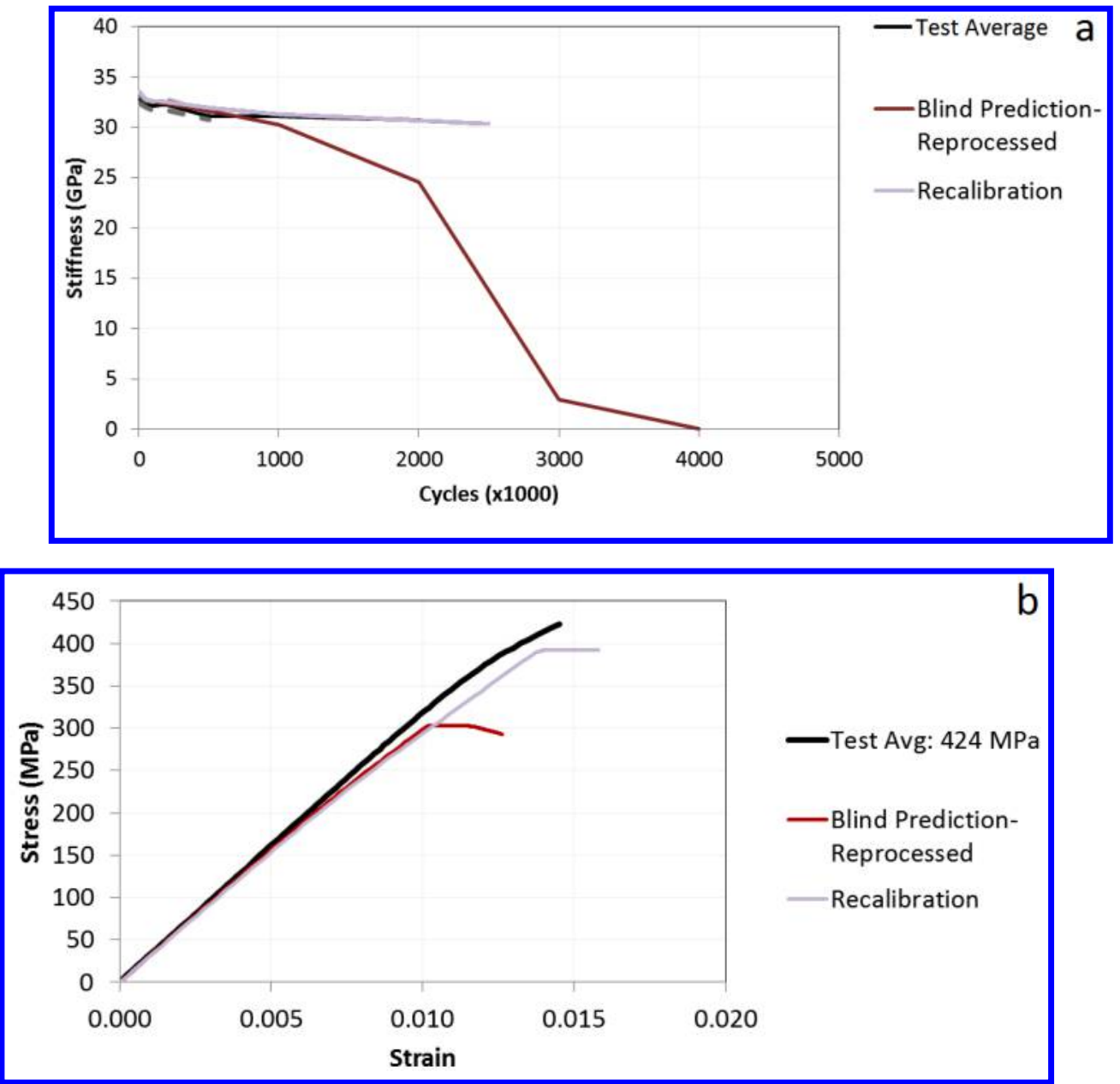


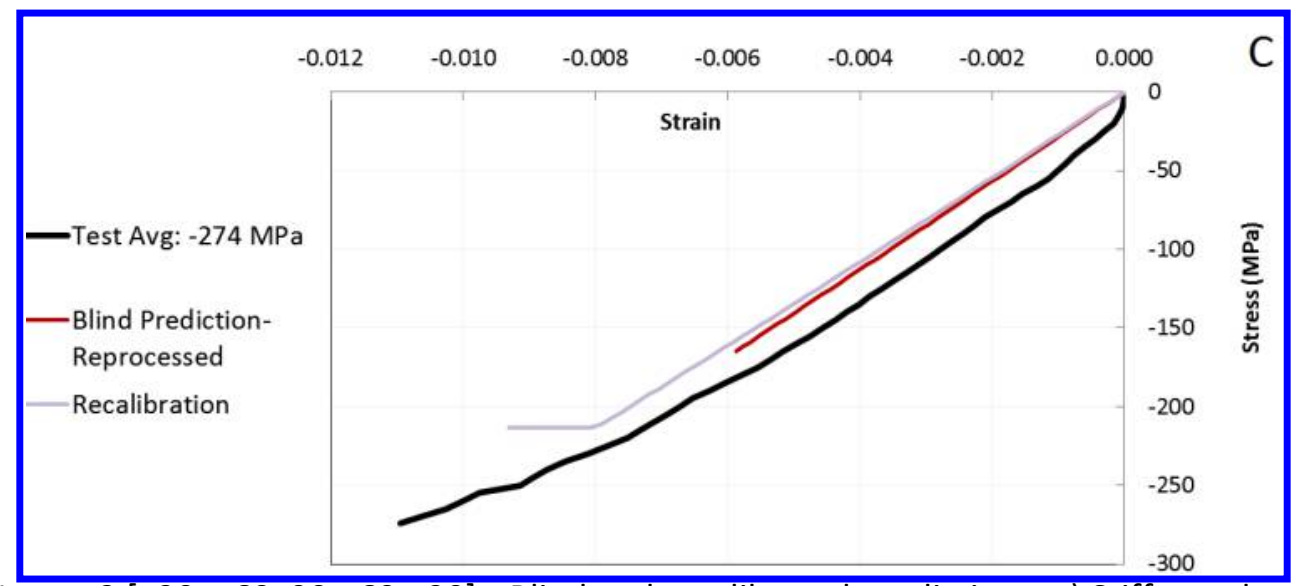

Figure 6: Layup $3[+30,+60,90,-60,-30]_{25}$ Blind and recalibrated predictions. a) Stiffness degradation as function of cycles, b) OHT residual strength after 200, 000 cycles, c) $\mathrm{OHC}$ residual strength after 200,000 cycles

The results, produced by only modifying the critical strain to failure, in the fiber (1-direction) are significantly better than those obtained during the blind prediction. The errors are reduced by almost a factor of two (see the errors given in Table 1 (Blind) and those given in Table 2 (after recalibration). This is expected since no experimental data was provided during the characterization portion of the study to ascertain the appropriate (length scale dependent) cyclic dependent longitudinal strain to failure, e.g., [0], [45/-45], or [0/90] for notched laminates. Consequently, a mere guess for the corresponding Weibull parameter, $m$, was made during the blind prediction stage.

Figure 7 shows damage contour plots of the individual plies under fatigue loading compared to individual X-rays provided by AFRL for Layup 3 obtained at different load cycles (blue represents no damage and green represents matrix failure). As can be expected, the propagation of damage in the 90 ply is much more evident than the other plies. Although the crack direction might not be fully aligned with the one observed in the X-rays, the approximate propagation direction is captured in almost all the plies. It can be seen that the fatigue model used was not able to capture the discrete cracking observed in the experiment. The reader is cautioned from making any definitive judgment on the predictive capability of the model, through comparison of these damage contour plots with the X-ray images, as there are numerous unknown factors involved in the interpretation of these images. According to AFRL, it is the nature of CT images to "bleed" across plies. Once a significant amount of damage forms, the dye penetrant used fills the voids and "glows" in a CT image. So for example in a 90 degree ply, there is some damage associated with the 90 ply plus some region of the interface and the adjacent plies, which is very hard to distinctly recognize and associate with the individual ply. 


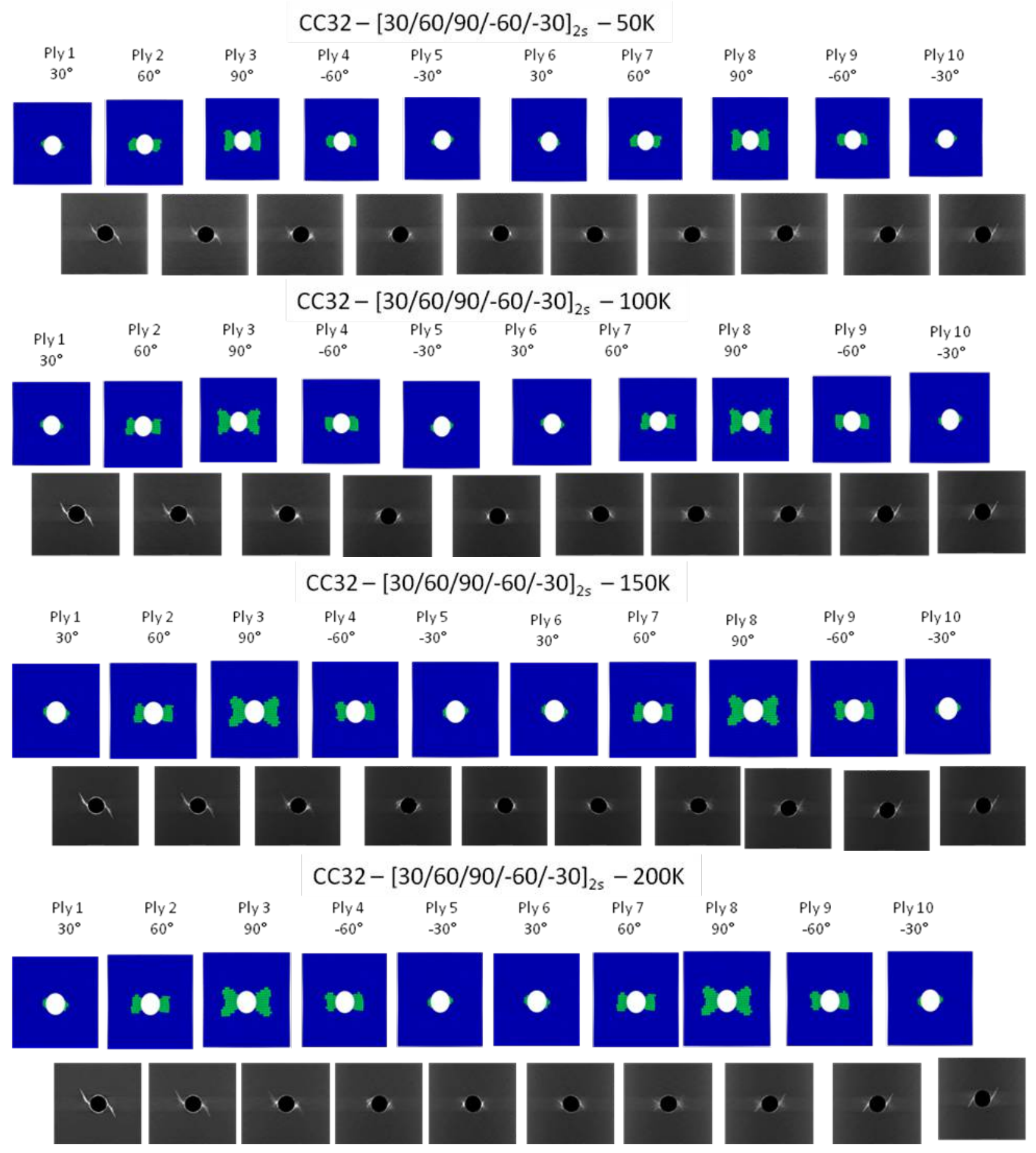

Figure 7: Layup $3[+30,+60,90,-60,-30]_{25}$ fatigue damage contour plots per ply corresponding to recalibrated predictions.

\section{Conclusion}

Fatigue and post-fatigue tension and compression response of three multidirectional layups were first blindly predicted and then recalibrated utilizing the ADEAL/EST model, however, only the detailed results for a specific Layup (Layup $3[+30,+60,90,-60,-30]_{2 S}$ ) was demonstrated here. A significant 
improvement was observed during recalibration by only modifying the critical strain to failure in the fiber (1-direction). Meanwhile, contour plots of the individual plies under fatigue loading compared to individual X-rays provided by AFRL for Layup 3 captures the approximate propagation direction is captured in almost all the plies, but were unable to capture the discrete nature of the cracking.

It should be mentioned that the input data provided for characterization was inadequate compared to the level of fidelity of the predictions requested. Providing simple unnotched characterization tests in which only cross head measurements are provided to glean cycle by cycle degradation of stiffness and then asking one to predict more accurate extensometer measurements of notched laminates is unrealistic because the coupon data offers no insight into what is occurring locally in the presence of a stress riser. Further, since the models are recalibrated based on the provided data for three given multidirectional layups, further blind predictions for other multidirectional layups might actually provide a better assessment of the predictive capability of the different numerical models. Finally further work that captures the mechanisms of failure in fatigue is needed in order to improve the EST based residual strength and ADEAL fatigue model presented here.

\section{Acknowledgement}

Anthony Waas would like to acknowledge funding by Lockheed Martin under AFRL Tech Scout 1: Damage Tolerant Design Principles (DTDP) program, Dr. Steve Engelstad, Principle Investigator Lockheed Martin. Dr. Stephen B. Clay, AFRL, program manager.

\section{References}

1. Arnold, S. M. and Kruch, S., (1994) "A Differential CDM Model For Fatigue of Unidirectional Metal Matrix Composites", Int. Jnl. of Damage Mechanics, Vol. 3, No. 2, pp.170-191.

-2. Arnold, S. M. and Castelli, M.G., (1994) "Continuum Based Theoretical and Experimental Studies in Deformation and Damage of MMCs at NASA Lewis: Progress and Trends, Composite Engineering, Vol. 4, No. 8, pp. 811-828.

3. Kruch, S. and Arnold, S. M., (1997) "Creep Damage and Creep-Fatigue Damage Interaction for Metal Matrix Composites "Applications of Continuum Damage Mechanics to Fatigue and Fracture, ASTM STP 1351, D.L. McDowell, Ed., American Society for Testing and Materials, pp. 728.

4. Wilt, T.E., Arnold, S. M.., and Saleeb, A.F., (1997) "A Coupled/Uncoupled Computational Scheme For Deformation and Fatigue Damage Analysis of Unidirectional MMC's", Applications of Continuum Damage Mechanics to Fatigue and Fracture, ASTM STP 1351, D.L. McDowell, Ed., American Society for Testing and Materials, pp. 65-82.

5. Bednarcyk, B. A. and Arnold, S. M.; "Fully Coupled Micro/Macro Deformation, Damage and Failure Prediction for SiC/Ti-15-3 Laminates", ASCE, Journal of Aerospace Engineering, Vol 15, No. 3, pp. 74-83, 2002.

6. Pineda E.J, Waas A.M., (2013) "Numerical Implementation of a Multiple-ISV ThermodynamicallyBased Work Potential Theory for Modeling Progressive Damage and Failure in Fiber-Reinforced Laminates", International Journal of Fracture, Vol 182, pp. 93-122

7. Aboudi, J., Arnold, S.M., Bednarcyk, B.A., Micromechanics of Composite Materials: A Generalized Multiscale Analysis Approach, Elsevier, Inc., 2013.

8. Engelstad, S.; AIR VEHICLE INTEGRATION AND TECHNOLOGY RESEARCH (AVIATR), Task Order 0037: Assessment, Quantification, and Benefits of Applying Damage Tolerant Design Principles to Advanced Composite Aircraft Structure, AFRL-RQ-WP-TR-2015-0068. 
9. Weibull, W. (1939). The phenomenon of rupture in solids. Proc., Royal Swedish Institute of Engineering Research (Ingenioersvetenskaps Akad. Handl.) 153, Stockholm, 1--55.

10. Cameron, K., Grimes-Ledesma, L., Phoenix, S., Murthy, P., Thesken, J., Saulsberry, R. “Orbiter Kevlar/Epoxy Composite Overwrapped Pressure Vessel Flight Rationale Technical Assessment Report", Vol I and II NASA NESC report, RP-07-34, April 2007. 
This article has been cited by:

1. Faisal Hasan Bhuiyan, Ray S. Fertig. A Physics-Based Combined Creep and Fatigue Methodology for Fiber-Reinforced Polymer Composites . [Citation] [PDF] [PDF Plus] 